Check for updates

Cite this: RSC Adv., 2019, 9, 18205

Received 16th February 2019

Accepted 19th May 2019

DOI: $10.1039 / c 9 r a 01202 h$

rsc.li/rsc-advances

\section{The effect of fermentation conditions on the structure and anti-tumor activity of polysaccharides from Cordyceps gunnii}

\author{
Xiao-Cui Liu, ${ }^{\text {ab }}$ Hongran Li, ${ }^{\text {acd }}$ Tong Kang, ${ }^{\text {acd }}$ Zhen-Yuan Zhu, (D) *acd Ying-Liang Liu, ${ }^{\text {e }}$ \\ Hui-Qing Sun ${ }^{\text {acd }}$ and Li-chao Pan ${ }^{\text {acd }}$
}

\begin{abstract}
This study investigates the effect of fermentation conditions on the structure and anti-tumor activity of intracellular polysaccharides (IPS) of Cordyceps gunnii (C. gunnii) in submerged fermentation. The environmental and nutritional conditions are determined in a shaker flask by a single factor test. The inhibition of IPS on S180 cells was as an optimization index. The results show that the optimal fermentation conditions of $\mathrm{C}$. gunnii are an initial $\mathrm{pH}$ value of 6 , a temperature of $25^{\circ} \mathrm{C}$, a rotation speed of $150 \mathrm{rpm}, 4 \%$ glucose, and $1.0 \%$ peptone. Under these conditions, the macro molecular weight $\left(M_{\mathrm{w}}\right)$ polysaccharide content and anti-tumor activity of IPS are significantly higher than that in the basal culture medium. GC, HPGPC, periodate oxidation-Smith degradation, NMR, and FT-IR determine the structural characteristics of CPS-JC and CPS-YH (pure IPS cultured in basal culture medium and optimal culture medium, respectively). The results indicate that CPS-JC is mainly composed of $\alpha$-D-glucans, whereas CPS-YH primarily contain $\alpha$-D-glucans with a trace amount of $\beta$-D-glucans.
\end{abstract}

\section{Introduction}

Cordyceps, a well-known and valued Chinese medicinal mushroom, has various biological and pharmacological functions such as anti-tumor, antioxidant, antiaging, anti-inflammatory, and immunity improving qualities., ${ }^{\mathbf{1} 2}$ Although C. gunnii and Cordyceps sinensis are different species, they display similar pharmacological activities, ${ }^{3,4}$ which is generally attributed to the presence of essential bioactive ingredients. These include polysaccharides, cordycepic acid, cordycepin, superoxide dismutase (SOD), and amino acids. ${ }^{5-9}$ Polysaccharides are the major bioactive constituents of Cordyceps, contributing to the maintenance of good health and anti-cancer activity. ${ }^{\mathbf{1 0 , 1 1}}$ During a previous study, a high-molecular-weight polysaccharide (CPS) obtained from the mycelia of $C$. gunnii, was isolated and purified. Results indicated that CPS significantly inhibited the growth of K562 cells in vitro. ${ }^{\mathbf{1 2}}$ Recently, fungus polysaccharides and polysaccharide-protein complexes have attracted attention

\footnotetext{
${ }^{a}$ State Key Laboratory of Food Nutrition and Safety, Tianjin University of Science and Technology, Tianjin 300457, PR China. E-mail: zhyuanzhu@tust.edu.cn; Fax: +86 22 60601437; Tel: +862260601437

${ }^{b}$ Key Laboratory of Food Bio-technology, School of Food and Bioengineering, Xihua University, Chengdu 610039, P. R. China

${ }^{c}$ Key Laboratory of Food Nutrition and Safety, Ministry of Education, Tianjin University of Science and Technology, Tianjin 300457, PR China

${ }^{d}$ College of Food Science and Biotechnology, Tianjin University of Science and Technology, Tianjin 300457, PR China

${ }^{e}$ School of Life Sciences, Guizhou Normal University, Guiyang, Guizhou 550001, China
}

in the biochemical and medical fields due to their anti-cancer effects. ${ }^{13-16}$

Since the fruiting bodies of wild $C$. gunnii are rare and expensive, an effective way to obtain large quantities of polysaccharides from Cordyceps is from the mycelium via liquid submerged fermentation. ${ }^{17,18}$ Fermentation is a useful way to produce bioactive substances with health-promoting properties. The fermentation conditions are crucial to the production of bioactive materials since they are directly related to secondary metabolite biosynthesis and cell proliferation. ${ }^{\mathbf{1 9 , 2 0}}$ Moreover, the fermentation conditions affect the activity of metabolites. Lin and Chiang reported that $C$. militaris samples cultured in Radix astragali displayed higher anti-tumor activity than those cultured in the synthetic medium. ${ }^{21}$ These results proved that the fermentation conditions played a significant role in the anti-tumor benefits of polysaccharides, and have an essential effect on structural variability. The research results of Kai et al. indicated that the monosaccharide composition of hetero-polysaccharides was highly dependent on the type of monosaccharide obtained from the fermentation medium. ${ }^{22}$ However, no notable studies currently exist involving the relationship between fermentation conditions, anti-tumor activity, and the structure of C. gunnii polysaccharides subjected to submerged.

Sarcoma 180 (S180) cell line is commonly used to establish the model of transplanted tumor in mice, because it lacks major histocompatibility complex molecules and is not easy to be rejected by recipient mice. And there is no specific requirement for the recipient mouse species. In addition, S180 ascites 
sarcoma cells are easier to subculture, and have higher survival rate. Therefore, researchers usually select S180 cells to investigate the anti-tumor activity of some substances from medicinal fungi. Shin et al. reported that the fruiting bodies polysaccharide of Paecilomyces japonica, a new type of Cordyceps spp., had antitumor activity on the S180 tumor cells. ${ }^{23}$ Huang et al. reported that the water-soluble polysaccharides (Pi-PCM0 to Pi-PCM2) from Poria cocos all exhibited anti-tumor activities in vivo (Sarcoma 180 solid tumor implanted 1 in BALB/c mice). ${ }^{24}$ Our previous research showed that polysaccharides extracted by different methods from C. gunnii mycelia have significant inhibition ability toward S180 sarcoma cells. ${ }^{25}$ MTT assay is widely used to detect the promoting cell proliferative activity and cytotoxicity of some natural active ingredients. This method is simple to operate, safe to use, low cost, and can achieve high detection sensitivity by controlling experimental conditions. Wei et al. reported that polysaccharide isolated from Dendrobium officinale had anti-tumor activity on HepG-2 cells by MTT assay. ${ }^{26}$ The growth inhibitory rates of the crude polysaccharides to human gastric cancer cells were evaluated in vitro by MTT assay. $^{3}$ The polysaccharide of strain FK1 was extracted from the mycelium free supernatant by boiling water method and evaluated for antitoxicity effect on two human cancer cell lines: HeLa cell line and lymphoblastoid cell line (LCL) by MTT method. ${ }^{27}$ The antitumor activities of Ganoderma lucidum polysaccharide to the human breast cancer cell (MDAMB-231) in vitro was evaluated by MTT assay. ${ }^{\mathbf{1 6}}$ Hence, in this paper we took the S180 ascites sarcoma cells and MTT assay to investigate the anti-tumor activity of polysaccharide from mycelium of $C$. gunnii in vitro. Different analysis types to determine anti-tumor activity such as morphological, genetic or molecular analysis need be further studied.

This study utilizes different environmental and nutritional conditions to cultivate $C$. gunnii and investigate the anti-tumor activity of the fermentation products. The purpose is to obtain the optimal culture medium suitable for the formation of polysaccharides possessing higher anti-tumor activity. Furthermore, the structural characteristics of CPS-JC and CPSYH were compared using a combination of chemical and instrumental analysis, such as acid hydrolysis, GC, HPGPC, periodate oxidation-Smith degradation, NMR, and FT-IR.

\section{Materials and methods}

\section{Materials}

Sarcoma 180 (S180) cells, obtained from the American type culture collection (ATCC), were prepared from peritoneal cavity of the tumor inoculated mice under aseptic conditions. The $C$. gunnii used in this study were obtained from the Key Laboratory of Food Nutrition and Safety (Ministry of Education, China), and the College of Food Science and Biotechnology (Tianjin University of Science and Technology, Tianjin, China). The strain of C. gunnii was maintained on Potato Dextrose Agar (PDA) slants, and was stored at $4{ }^{\circ} \mathrm{C}$ after the slant was incubated at $25^{\circ} \mathrm{C}$ for $7 \mathrm{~d}$. The S180 cells were maintained in RPMI Medium 1640 supplemented with 10\% fetal bovine serum, 100 $\mu \mathrm{g} \mathrm{mL} \mathrm{m}^{-1}$ streptomycin, and $100 \mathrm{mU} \mathrm{L}{ }^{-1}$ penicillin at $37^{\circ} \mathrm{C}$ in a humidified atmosphere of $5 \% \mathrm{CO}_{2}$.

The standard monosaccharides (D-glucose, D-xylose, Dgalactose, L-rhamnose, D-mannose, and D-arabinose), Sephadex G-100 and 3-(4,5-dimethylthiazol-2-yl)-2,5-diphenyl-tetrazolium bromide (MTT) were purchased from the Sigma Chemical Co. (St. Louis, MO, USA). All other chemicals used in this study were of analytical grade.

\section{Preparation of the mycelium}

The mycelium of $C$. gunnii was activated by culturing the sample on a PDA plate at $25{ }^{\circ} \mathrm{C}$ for $7 \mathrm{~d}$. Following this process, the sample was transferred to the seed culture medium by punching out agar discs of approximately $5 \mathrm{~mm}$ in diameter from the culture grown on the PDA plates. The seed liquid was grown in a $250 \mathrm{~mL}$ flask containing $100 \mathrm{~mL}$ of seed culture medium (sucrose $20 \mathrm{~g} \mathrm{~L}^{-1}$, peptone $10 \mathrm{~g} \mathrm{~L}^{-1}, \mathrm{KH}_{2} \mathrm{PO}_{4} 3 \mathrm{~g} \mathrm{~L}^{-1}, \mathrm{MgSO}_{4}$ $\cdot 7 \mathrm{H}_{2} \mathrm{O} 1.5 \mathrm{~g} \mathrm{~L}^{-1}$, distilled water, initially natural $\mathrm{pH}$ ) at $25^{\circ} \mathrm{C}$ on a rotary shaker incubator at $150 \mathrm{rpm}$ for $3 \mathrm{~d}$.

The flask culture experiments were conducted in $250 \mathrm{~mL}$ flasks containing $100 \mathrm{~mL}$ of the basal culture medium (sucrose $40 \mathrm{~g} \mathrm{~L}^{-1}$, peptone $5 \mathrm{~g} \mathrm{~L}^{-1}, \mathrm{KH}_{2} \mathrm{PO}_{4} 3 \mathrm{~g} \mathrm{~L}{ }^{-1}, \mathrm{MgSO}_{4} \cdot 7 \mathrm{H}_{2} \mathrm{O} 1.5 \mathrm{~g}$ $\mathrm{L}^{-1}$, distilled water, initially natural $\mathrm{pH}$ ) after being inoculated with $8 \%(\mathrm{v} / \mathrm{v})$ of the seed liquid.

A single factor-screening test was used to investigate each factor of the optimal medium, ${ }^{28}$ and to ascertain the optimal culture conditions (environmental conditions and nutrition requirements) for determining the IPS displaying the highest anti-tumor activity. First, the anti-tumor activity of IPS was considered as optimization indexes to select the optimal environmental conditions including initial $\mathrm{pH}$ value, temperature, and rotation speed. Then, based on these established environmental conditions, the anti-tumor activity of IPS was used as an additional indicator to select the optimal nutritional requirements including carbon source types, nitrogen source types, as well as their concentration levels. Finally, the mycelium of $C$. gunnii was cultured in the basal culture medium and the optimal medium, respectively. The cultured mycelia from one sample were freeze-dried after being separated from the medium and thoroughly rinsed with a large amount of distilled water. ${ }^{29}$ Moreover, the crude polysaccharides extracted from the two types of mycelium were named JC and YH, respectively.

\section{The extraction and purification of crude IPS}

IPS were extracted from dry mycelial powder $(400 \mathrm{mg})$ by suspending the mycelia in $8 \mathrm{~mL}$ distilled water at $80{ }^{\circ} \mathrm{C}$ for $2 \mathrm{~h}$ and repeating twice. The extracts were gathered in a container and added to four volumes of $95 \%(\mathrm{v} / \mathrm{v})$ ethanol to precipitate overnight at $4{ }^{\circ} \mathrm{C}$. Following this process, the samples were centrifuged at $4500 \times g$ for $20 \mathrm{~min}$, and the supernatant fluid was collected. The extract was concentrated to a volume of $6 \mathrm{~mL}$ by being exposed to reduced pressure. The concentrated solution was deproteinated using the Sevag method and repeating the process seven times. The crude polysaccharides (JC and $\mathrm{YH}$ ) were subjected to gel filtration on a column $(30 \mathrm{~cm} \times 3 \mathrm{~cm})$ of Sephadex G-100, eluted with deionized water at a flow rate of 0.3 
$\mathrm{mL} \min ^{-1}$, and monitored using the phenol-sulfuric acid method.

\section{Anti-tumor activity analysis}

The anti-tumor activity of the IPS in S180 ascites tumor cells was evaluated in vitro with an MTT assay. The S180 cells were seeded at a concentration of $1 \times 10^{5}$ cells per $\mathrm{mL}$ in a volume of $100 \mu \mathrm{L}$ in 96-well plates. Cells were incubated with the IPS samples at respective concentrations of $25 \mu \mathrm{g} \mathrm{mL} L^{-1}, 50 \mu \mathrm{g} \mathrm{mL}{ }^{-1}, 100 \mu \mathrm{g}$ $\mathrm{mL}^{-1}, 200 \mu \mathrm{g} \mathrm{mL}{ }^{-1}$, and $400 \mu \mathrm{g} \mathrm{mL}^{-1}$. After $20 \mathrm{~h}, 44 \mathrm{~h}, 68 \mathrm{~h}$, respectively, $20 \mu \mathrm{L}$ of $5 \mathrm{mg} \mathrm{mL}{ }^{-1}$ of MTT was added to each well and incubated for another $4 \mathrm{~h}$. The culture media were subsequently removed, and $100 \mu \mathrm{L}$ of dimethyl sulfoxide (DMSO) was added to each well. A micro-plate ELISA reader was used to detect absorbance length at $490 \mathrm{~nm} .^{30}$ The inhibition ratio of S180 cell proliferation was calculated as follows:

$$
\phi=\frac{\mathrm{OD}_{\mathrm{a}}-\mathrm{OD}_{\mathrm{b}}}{\mathrm{OD}_{\mathrm{a}}} \times 100 \%
$$

$\mathrm{OD}_{\mathrm{a}}$ is the absorbance value of the negative control group, and $\mathrm{OD}_{\mathrm{b}}$ is that of the sample group.

\section{Determination of $M_{\mathrm{w}}$ distribution}

The $M_{\mathrm{w}}$ distribution of IPS was determined by HPGPC with an Agilent-1200 instrument (Agilent, USA), equipped with a TSK gel G4000PWxl column $(7.8 \mathrm{~mm} \times 300 \mathrm{~mm}$, and a column temperature $30{ }^{\circ} \mathrm{C}$ ). The sample was eluted with deionized water

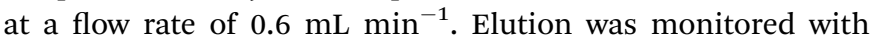
a Refractive Index Detector (RID), at a detecting temperature of $35{ }^{\circ} \mathrm{C}$. The standard curve was established using T-series Dextran as the standards (T-10, T-40, T-70, T-500, and T-2000). The $M_{\mathrm{w}}$ of each composition was calculated by comparison with the retention time of the monosaccharide reference standard. Based on the peak area values, the relative percentage content of each fraction could be determined. ${ }^{31}$

\section{Fourier transform infrared (FT-IR) analysis}

The FT-IR spectrum analysis was performed on a $1 \mathrm{~mm}$ pellet by pressing $1 \mathrm{mg}$ of polysaccharide grinding mixture with $150 \mathrm{mg}$ of dried $\mathrm{KBr}$. The spectra length was recorded at the absorbance mode in the region of $4000-400 \mathrm{~cm}^{-1}$ on an FT-IR spectrophotometer (VECTOR-22).

\section{Nuclear magnetic resonance (NMR) spectroscopy}

${ }^{1} \mathrm{H}$ NMR and ${ }^{13} \mathrm{C}$ NMR spectra were recorded on a Bruker spectrometer $(600 \mathrm{MHz})$ at a probe temperature of $298 \mathrm{~K}$. Before the analysis, the samples were exchanged twice with $\mathrm{D}_{2} \mathrm{O}$ while freeze-drying.

\section{Monosaccharide analysis}

The polysaccharides were hydrolyzed with $2 \mathrm{M}$ trifluoroacetic acid (TFA) at $110{ }^{\circ} \mathrm{C}$ in a sealed tube for $3 \mathrm{~h}$. One part of the hydrolysate was analyzed by Thin-Layer Chromatography (TLC) was used to analyze one part of the hydrolysate. The other part was acetylated and analyzed using gas chromatography (GC) after the TFA was removed. D-Glucose, D-xylose, D-galactose, Lrhamnose, D-mannose, and D-arabinose were derived as standards.

\section{Periodate oxidation and Smith degradation analysis}

A $5.0 \mathrm{mg}$ volume of the samples were dissolved in a $25 \mathrm{~mL}$ solution containing $0.03 \mathrm{M} \mathrm{NaIO}_{4}$, and kept in darkness at $4{ }^{\circ} \mathrm{C}$. A $0.1 \mathrm{~mL}$ volume of the solution was drawn every $8 \mathrm{~h}$, diluted to $25 \mathrm{~mL}$ with distilled water, and detected with a UV spectrophotometer at $223 \mathrm{~nm}$. The process completed after $96 \mathrm{~h}$, and ethylene glycol $(0.2 \mathrm{~mL})$ was added to the solution to quench the reaction. Consumption of $\mathrm{NaIO}_{4}$ was measured using the spectrophotometric method, and the production of formic acid was determined by titration with $0.01 \mathrm{M} \mathrm{NaOH}$. The reaction mixture was then dialyzed in distilled water, and the nondialysate was reduced with $\mathrm{NaBH}_{4}(30 \mathrm{mg})$ for $12 \mathrm{~h} .{ }^{32}$ Finally, the $\mathrm{pH}$ was adjusted to 5.0-6.0, the solution was dialyzed, the non-dialysate was lyophilized, and then hydrolyzed with $2 \mathrm{~mL}$ of $2 \mathrm{M}$ TFA at $110{ }^{\circ} \mathrm{C}$ for $3 \mathrm{~h}$. The subsequent hydrolysate was analyzed by GC.

\section{Statistical analysis}

Data were expressed as means \pm SD at least three independent experiments. Data in all the bioassays were statistically evaluated by analysis of variance. ${ }^{*} p<0.05,{ }^{* *} p<0.01$.

\section{Results and discussion}

\section{The effect of different fermentation conditions on the anti- tumor activity and $M_{\mathrm{w}}$ distribution of crude IPS in C. gunnii}

The $M_{\mathrm{w}}$ of the polysaccharides were determined by a universal calibration curve using Dextran as standards. The calibration curve of the Dextran standards was plotted as the $M_{\mathrm{w}}$ on a log scale versus the retention time. ${ }^{33}$ The regression equation was $\lg M_{\mathrm{w}}=9.3621-0.3481 t_{\mathrm{R}}, R^{2}=0.9899\left(M_{\mathrm{w}}\right.$ : weight-average molar mass, Da; $t_{\mathrm{R}}$ : the retention time, min). As shown in Table 1, the HPGPC profile of each crude IPS had three peaks, indicating that they contained three types of polysaccharides, respectively. The relative content of three fractions displayed significant differences, while the first fraction exhibited the highest content. ${ }^{10}$ On the basis of the theory of the macromolecular polysaccharide appeared firstly in HPGPC chromatogram, it indicated that all samples were main containing macro $M_{\mathrm{w}}$ polysaccharide (macro $M_{\mathrm{w}}$ : molecular weight approximately $3 \times 10^{6} \mathrm{Da}$ ), with a trace amount of medium $M_{\mathrm{w}}$ (medium $M_{\mathrm{w}}$ : $M_{\mathrm{w}}$ approximately $4 \times 10^{5} \mathrm{Da}$ ) and low $M_{\mathrm{w}}$ (low $M_{\mathrm{w}}: M_{\mathrm{w}}$ approximately $1 \times 10^{4} \mathrm{Da}$ ) polysaccharide.

A medium $\mathrm{pH}$ level has a seemingly significant impact on cell membrane function, cell morphology and structure, as well as the uptake of various nutrients, and product biosynthesis in all the environmental factors. ${ }^{34}$ As shown in Table 1, the highest macro $M_{\mathrm{w}}$ polysaccharide of IPS reached $82.717 \%$ when the $\mathrm{pH}$ value of fermentation medium was 6.0, and the IPS production reached $(95.47 \pm 2.92 \mathrm{mg} / 100 \mathrm{~mL})$ and was higher $(10-20) \mathrm{mg} /$ $100 \mathrm{~mL}$ than other samples in the $\mathrm{pH}$ group. Additionally, the IPS cultured at $\mathrm{pH} 6.0$ exhibited a higher inhibition ratio at 
Table 1 Effects of different fermentation conditions on the molecular weight distribution and production of IPS in submerged cultivation of Cordyceps gunnii ${ }^{a}$

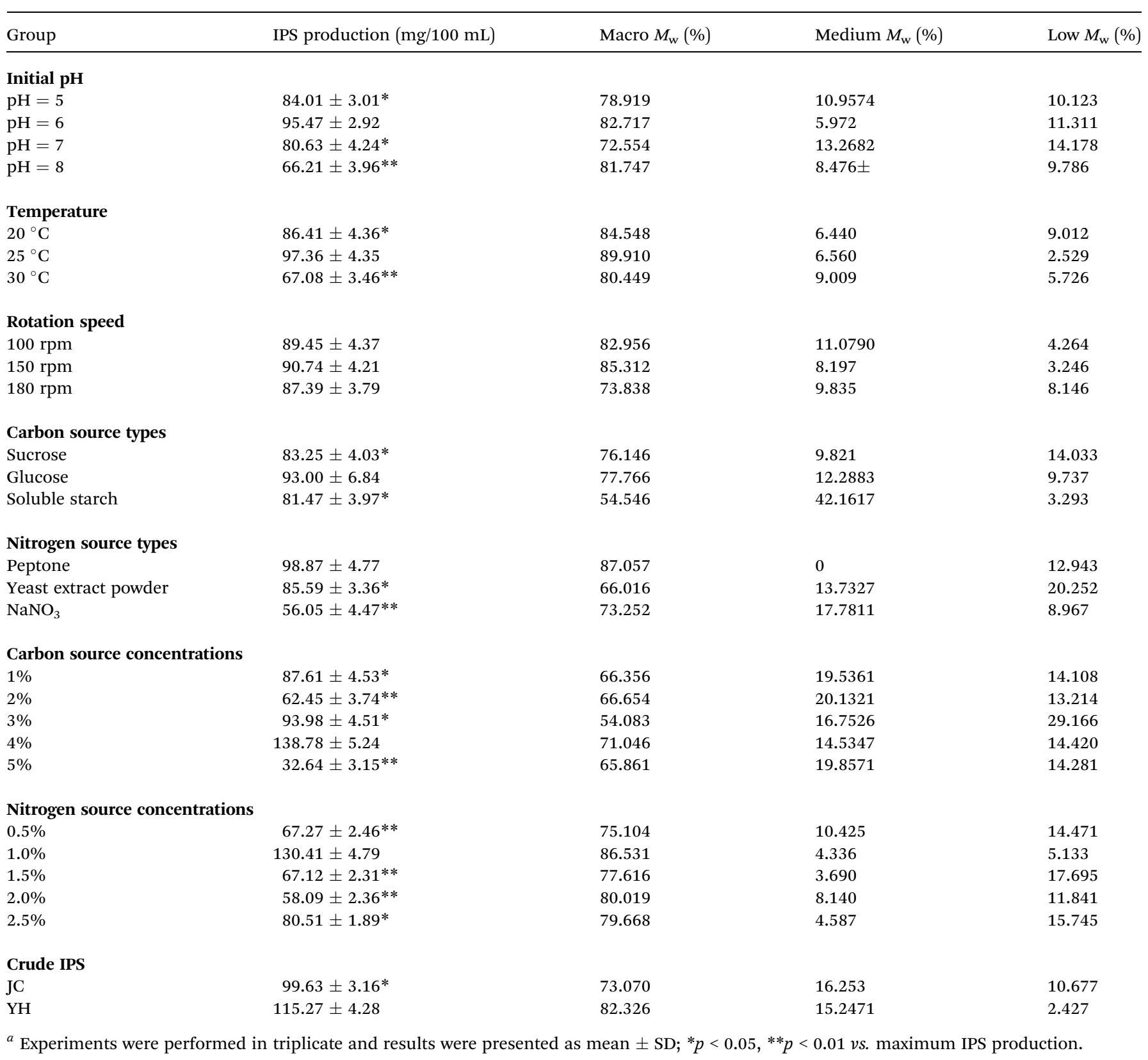

about $60 \%$ in the $\mathrm{S} 180$ cells at a concentration of $400 \mu \mathrm{g} \mathrm{mL}$ (Fig. 1A). The results indicated that $\mathrm{pH} 6.0$ was the optimal $\mathrm{pH}$ value to promote the initial growth of IPS displaying the highest anti-tumor activity.

S180 cells, were exposed to three different fermentation temperatures $\left(20^{\circ} \mathrm{C}, 25^{\circ} \mathrm{C}\right.$, and $\left.30^{\circ} \mathrm{C}\right)$ in shake flask cultures to evaluate anti-tumor activity (Fig. 1B). There is no significant difference of inhibition ratio between $25{ }^{\circ} \mathrm{C}$ and $30{ }^{\circ} \mathrm{C}$ at the concentration of $200 \mu \mathrm{g} \mathrm{mL}{ }^{-1}$ and $400 \mu \mathrm{g} \mathrm{mL}{ }^{-1}$. However, the IPS obtained a relatively higher inhibition ratio at $25{ }^{\circ} \mathrm{C}$ than that at $30{ }^{\circ} \mathrm{C}$ for $400 \mu \mathrm{g} \mathrm{mL}{ }^{-1}$ concentration. At the same culture temperature, macro $M_{\mathrm{w}}$ polysaccharide content and IPS production of C. gunnii both reached the highest level at $89.91 \%$ and $97.36 \pm 4.35 \mathrm{mg} / 100 \mathrm{~mL}$, respectively (Table 1 ). This result corresponded with the findings of existing literature that the optimal cultivation temperature for Cordyceps mycelia in a submerged fermentation culture medium appeared to be $25{ }^{\circ} \mathrm{C}^{21}$

It is well-known that oxygen supply is closely related to the formation and accumulation of bioactive metabolites in medicinal fungal submerged cultivation processes. ${ }^{35}$ Rotation speeds of the shaking table can directly affect the oxygen capacity of the fermented liquid. Therefore, the effects of rotation speeds (100 rpm, $150 \mathrm{rpm}$, and $180 \mathrm{rpm}$ ) on the IPS production and activity were also investigated. As shown in Table 1, the IPS production and macro $M_{\mathrm{w}}$ polysaccharide 


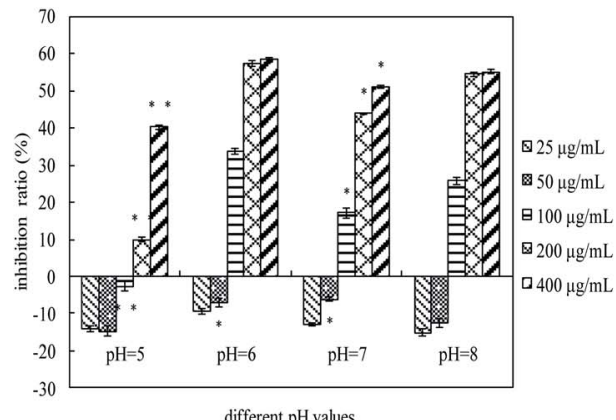

A

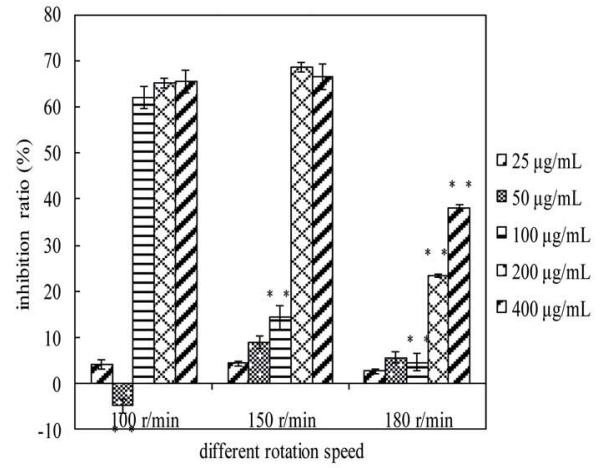

$\mathrm{C}$

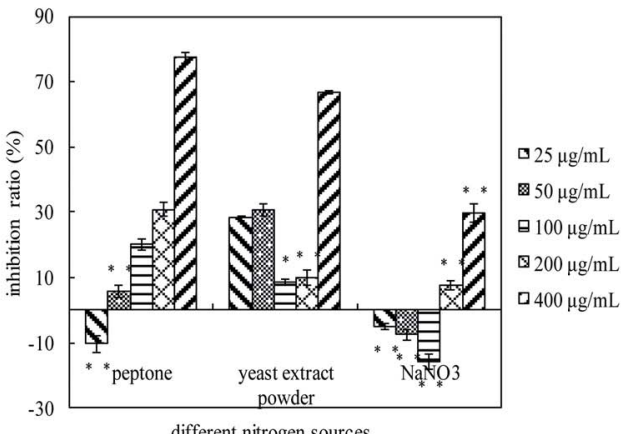

E

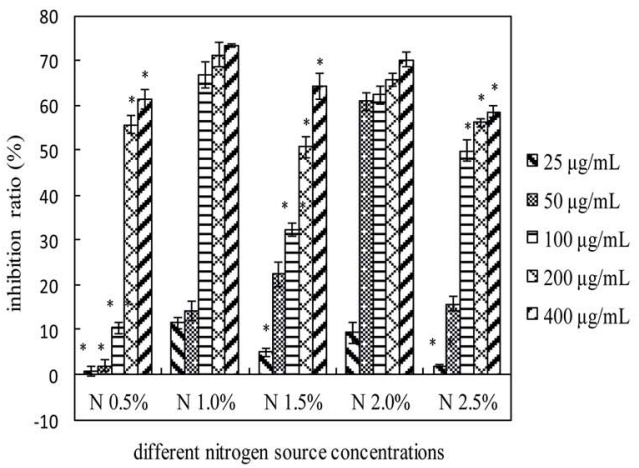



B



D



F

G

Fig. 1 The growth inhibitory effect of polysaccharide on S180 cell at different concentrations $(n=6)$ under different pH values (A), temperatures $(B)$, rotation speed (C), carbon sources (D), nitrogen sources (E), carbon source concentrations (F), and nitrogen source concentrations (G). * $p<$ $0.05, * * p<0.01$ vs. the highest inhibition rate at the same concentration of different groups.

content at a rotation speed of $150 \mathrm{rpm}$ exhibited a relatively higher level than that at the other two rotation speeds. Additionally, anti-tumor activity of IPS reached the highest levels at a rotation speed of $150 \mathrm{rpm}$ for $200 \mu \mathrm{g} \mathrm{mL} L^{-1}$ and $400 \mu \mathrm{g} \mathrm{mL}$ concentrations (Fig. 1C). In comparison with other rotation speeds, $150 \mathrm{rpm}$ was a good candidate for the rotation speed for 
its suitable dissolved oxygen amount and low machine loss. Therefore, $150 \mathrm{rpm}$ was the best choice of rotation speed.

Carbon sources exerted considerable influence on the production and compositions of polysaccharides in fungi. ${ }^{36}$ To determine a suitable carbon source for the production of $C$. gunnii with a higher anti-tumor activity IPS, various carbon sources such as sucrose, glucose, and soluble starch were individually utilized at a concentration of $40 \mathrm{~g} \mathrm{~L}^{-1}$ in the basal medium. As shown in Table 1, the maximum IPS production $(93.00 \pm 6.84 \mathrm{mg} / 100 \mathrm{~mL})$ was obtained by using glucose as the carbon source in the basic medium, and was significantly higher than sucrose and soluble starch $\left({ }^{*} p<0.05\right)$. This result does not correspond to the nutritional requirements of other species of Cordyceps in submerged cultures. Hsieh et al. reported that sucrose could significantly affect the mycelium growth of C. sinensis. ${ }^{37}$ The fact that carbon sources improved the production of polysaccharides might be attributed to energy provision for the fungi growth, and the accumulation of polysaccharides. Moreover, the macro $M_{\mathrm{w}}$ polysaccharide content and anti-tumor activity of IPS (Table 1 and Fig. 1D) in the glucose group appeared to be higher than in other carbon source groups. Moreover, the promoting effect was evident in S180 cell growth when soluble starch was used as the carbon source. The reasons might be that the starch was not completely dissolved in the medium. Compared with other carbon sources, glucose was a good candidate for the carbon source due to its ease-of-use and low cost. Therefore, glucose was selected as the primary carbon source for subsequent experiments.

The nitrogen sources are essential factors for mycelium growth and polysaccharide production. ${ }^{38}$ The results (Table 1) showed that the maximum IPS production $(98.87 \pm 4.77 \mathrm{mg} / 100$ $\mathrm{mL}$ ) and macro $M_{\mathrm{w}}$ polysaccharide content $87.057 \%$ were obtained by using peptone as a nitrogen source. The effects of nitrogen sources on the anti-tumor activity of IPS in C. gunnii during submerged cultivation are shown in Fig. 1E. IPS cultured in an inorganic nitrogen source $\left(\mathrm{NaNO}_{3}\right)$ medium exhibited relatively lower anti-tumor activity than the samples cultured in an organic nitrogen source (peptone, yeast extract powder) medium. This result is consistent with previous suggestions that most basidiomycetes prefer complex organic nitrogen sources to promote their growth in submerged cultures, ${ }^{39}$ and possibly for certain essential amino acid(s) that were barely synthesized from inorganic nitrogen sources in the cultivation of active substances. ${ }^{40}$ Peptone yielded the best IPS with higher anti-tumor activity compared with all other organic sources.

Additionally, in order to obtain the optimal carbon source and nitrogen source concentration, C. gunnii was cultivated with various levels of glucose (i.e. $1 \%, 2 \%, 3 \%, 4 \%$, and $5 \%$, w/v), and peptone concentrations (i.e. $0.5 \%, 1.0 \%, 1.5 \%, 2.0 \%$ and $2.5 \%$, $\mathrm{w} / \mathrm{v})$. As shown in Table 1, the highest IPS production and macro $M_{\mathrm{w}}$ polysaccharide content were obtained at $4 \%$ glucose (138.78 $\pm 5.24 \mathrm{mg} / 100 \mathrm{~mL} ; 71.046 \%)$ and $1.0 \%$ peptone $(130.41 \pm 4.79$ mg/100 mL; 86.531\%), respectively. As shown in Fig. 1F and G, considering the four different concentration levels, IPS exhibited significantly higher anti-tumor activity when the glucose concentration was at $4 \%$, and the nitrogen source concentration was at $1.0 \%$. These results indicated that a higher glucose concentration and a lower peptone concentration were beneficial to the production and anti-tumor activity of IPS. These findings correspond with previous studies that high concentrations of glucose were beneficial to the mycelial formation. ${ }^{41}$

In this study, the polysaccharide at lower concentrations exhibited proliferative effect on S180 cells. The result was coincided with the report in the literature that extracellular polysaccharide (Pi-PCM0) from Poria cocos mycelia exhibit proliferative effect on human MCF-7 cells at the lower concentration of $50 \mu \mathrm{g} \mathrm{mL}{ }^{-1}{ }^{42}$ Peng et al. reported that the heteropolysaccharide coded as EPF1 was obtained from the crude extracellular polysaccharide of Ganoderma tsugae mycelium also exhibit proliferative effect on Sarcoma 180 cells at the lower concentration of $0.005 \mathrm{~g} \mathrm{~L}^{-1} .43$ It implied that some polysaccharide at lower concentration did not have toxicity to tumor cells, which need to be explained by further research.

Therefore, all the fermentation conditions (including environmental conditions and nutritional conditions) significantly affected the production, $M_{\mathrm{w}}$ distribution, and anti-tumor activity of IPS in C. gunnii in submerged fermentation. Wang et al. reported that the cytokine nitric oxide (NO) can inhibit of a variety of tumors, and polysaccharides with a $M_{\mathrm{w}}$ above 100 $\mathrm{kDa}$ could significantly promote the release of NO. ${ }^{44}$ Therefore, the fermentation conditions influenced the $M_{\mathrm{w}}$ of polysaccharides, and related to the anti-tumor activity. Moreover, the results of this study indicated that polysaccharides with a larger $M_{\mathrm{w}}$ displayed higher anti-tumor activity when they were cultured in different fermentation mediums.

Considering all the available results, the optimal culture process of C. gunnii was determined at an initial $\mathrm{pH}$ value of 6 , a temperature of $25{ }^{\circ} \mathrm{C}$, a rotation speed of $150 \mathrm{rpm}, 4 \%$ glucose, and $1.0 \%$ peptone. The crude IPS fermented in the optimized medium was defined as $\mathrm{YH}$, while the crude IPS fermented in the basal medium was defined as JC.

\section{Isolation and purification of JC and $\mathrm{YH}$}

JC and YH, were obtained from the mycelium of C. gunnii using hot water extraction and ethanol precipitation. As shown in Table 1, the IPS production of JC and YH were $99.63 \pm 3.16 \mathrm{mg} /$ $100 \mathrm{~mL}$. and $115.27 \pm 4.28 \mathrm{mg} / 100 \mathrm{~mL}$, respectively. The total sugar content of JC and $\mathrm{YH}$ were $42.30 \%$ and $54.07 \%$, respectively, and was detected using a phenol-sulfuric acid assay as $\mathrm{D}^{-}$ glucose equivalents. Additionally, JC and YH were separated with Sephadex G-100 column chromatography, and the elution curve is shown in Fig. 2A and B, respectively. The main fraction (CPS-JC and CPS-YH) was collected and lyophilized for further study relating to structure and anti-tumor activity. The HPGPC profile demonstrated that CPS-JC and CPS-YH (Fig. 2C and D) produced a single and symmetrical peak, revealing that both were homogeneous polysaccharides. Based on the equation of the standard curve generated by various Dextran standards and the retention time of CPS-JC and CPS-YH, the average $M_{\mathrm{w}}$ was estimated to be $8.8 \times 10^{5} \mathrm{Da}$ and $12.68 \times 10^{5} \mathrm{Da}$, respectively. The specific optical rotation of CPS-JC and CPS-YH were determined to be $+95^{\circ}$ and $-35^{\circ}$, respectively. The $\mathrm{UV}$ absorption spectra of CPS-JC and CPS-YH exhibited no absorption at 280 




A
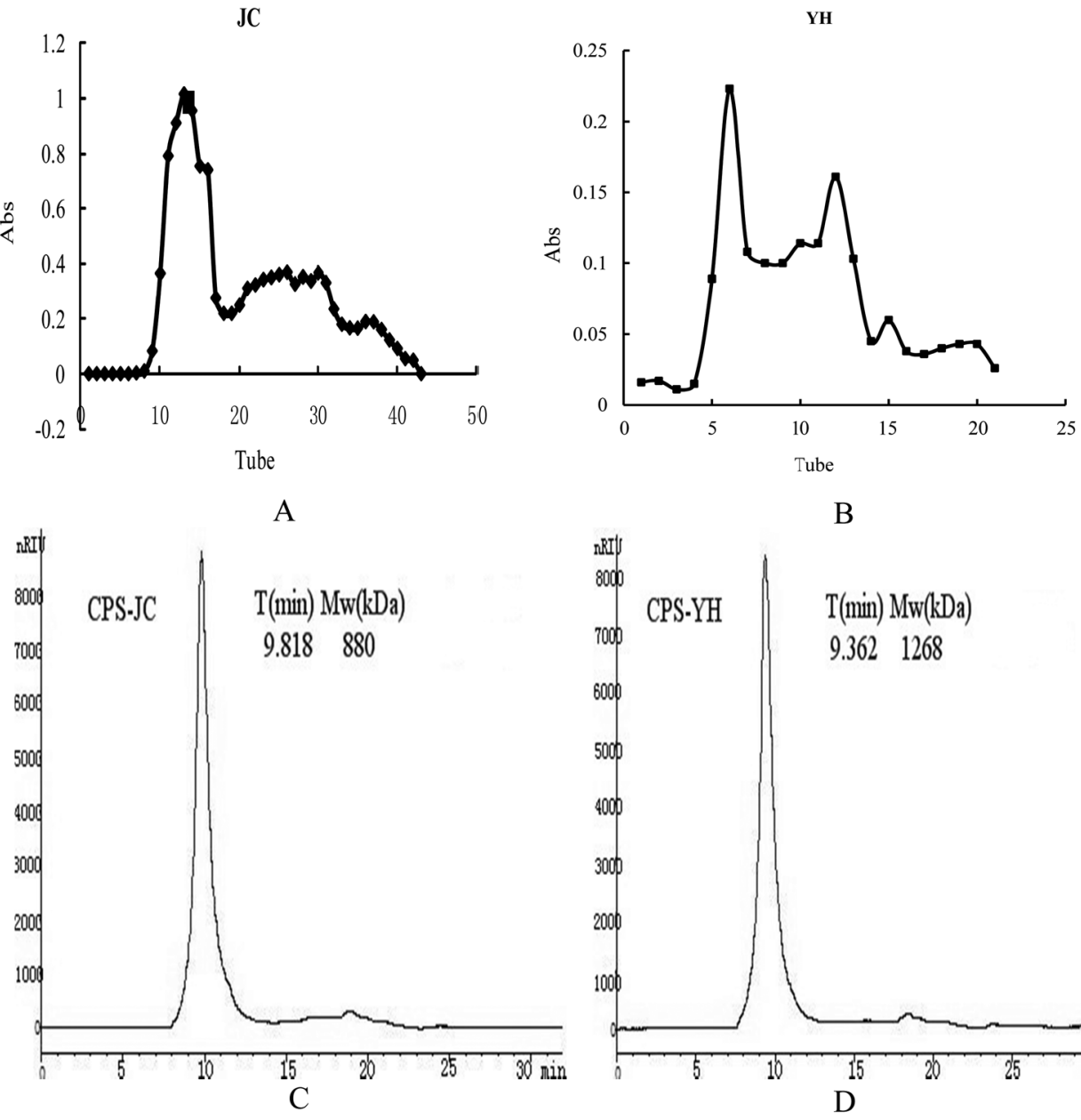

B


Fig. 2 Elution profiles of JC (A) and YH (B) in Sephadex G-100 column chromatography; HPGPC profiles of CPS-JC (C) and CPS-YH (D); the growth inhibitory effect of CPS-JC and CPS-YH (E) on S180 cell at different concentrations $(n=6)$. ${ }^{*} p<0.05, * * p<0.01$, compared to the control.

and $260 \mathrm{~nm}$, indicating the absence of proteins and nucleic acids from the polysaccharides. The total sugar content of CPSJC and CPS-YH were $64.93 \%$ and $75.01 \%$, respectively.
Interestingly, the measured value of polysaccharide content was lower than the theoretical value, which might be attributed to polyol-water crystallization water. 


\section{The anti-tumor activity in vitro of CPS-JC and CPS-YH}

In this study, S180 cells were cultured with different concentrations of CPS-JC and CPS-YH for various periods. As shown in Fig. 2E, at a concentration of $400 \mu \mathrm{g} \mathrm{mL} \mathrm{m}^{-1}$, CPS-YH exhibited a substantially higher inhibition ratio at about $>75 \%$, while CPS-JC displayed an inhibition ratio about $70 \%$. At concentrations of $200 \mu \mathrm{g} \mathrm{mL}{ }^{-1}$ and $100 \mu \mathrm{g} \mathrm{mL}{ }^{-1}$, the activity presented a significant difference $(* p<0.05)$; the inhibition ratio reached about $70 \%$ in CPS-YH, while it reached only $40 \%$ in CPS-JC. At a concentration of $50 \mu \mathrm{g} \mathrm{mL}{ }^{-1}$, the inhibition ratios in the $\mathrm{S} 180$ cells were $24.63 \%$ and $54.66 \%$ of CPS-JC and CPS-YH, respectively. Additionally, the inhibition ratio of CPS-YH on S180 cells reached $65.18 \%$ and was significantly higher than CPS-JC when exposed to the same conditions with $25 \mu \mathrm{g} \mathrm{mL}{ }^{-1}$.



A

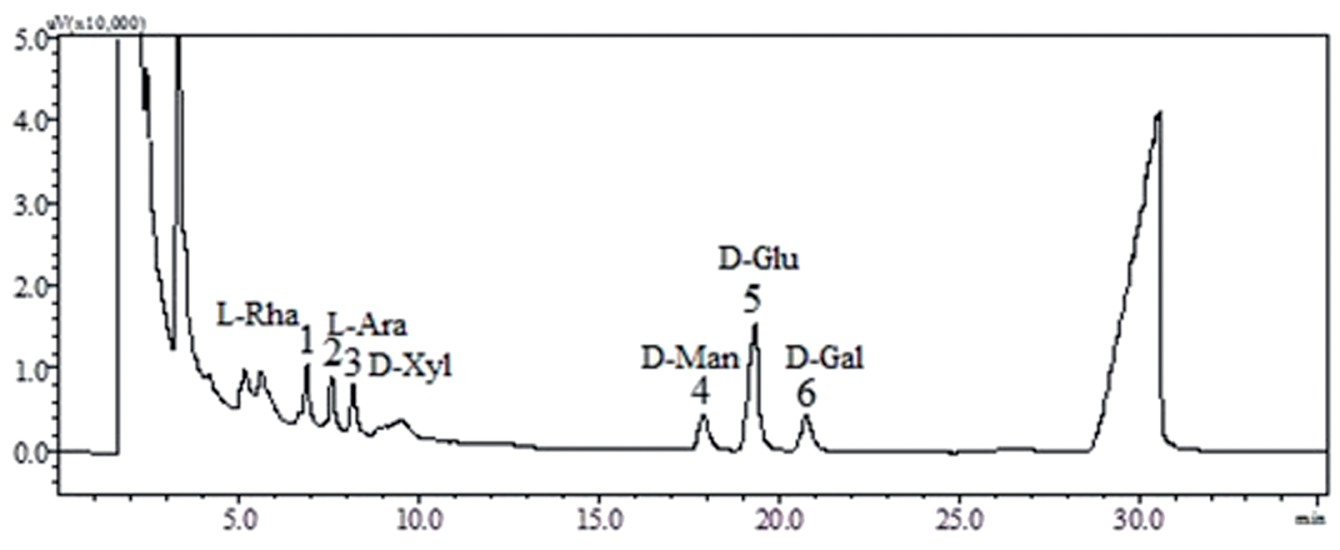

B

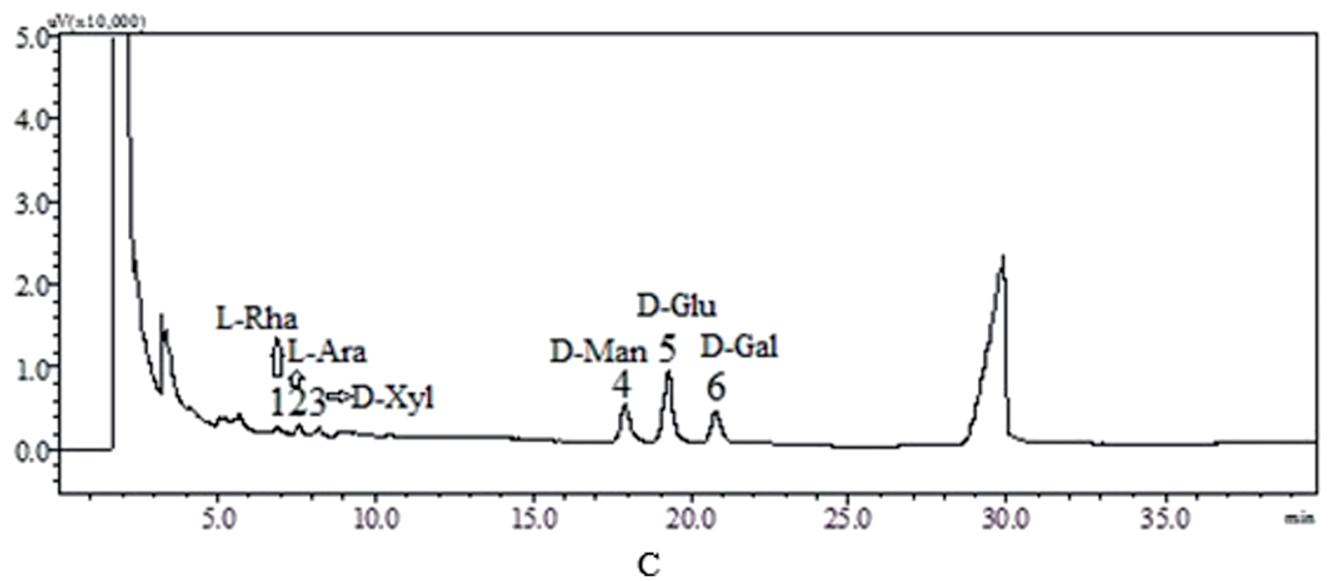

Fig. 3 Thin-Layer Chromatography (TLC) of CPS-JC and CPS-YH (A) after acid hydrolysis; the gas chromatography (GC) of derivatives CPS-JC (B) and CPS-YH (C). 


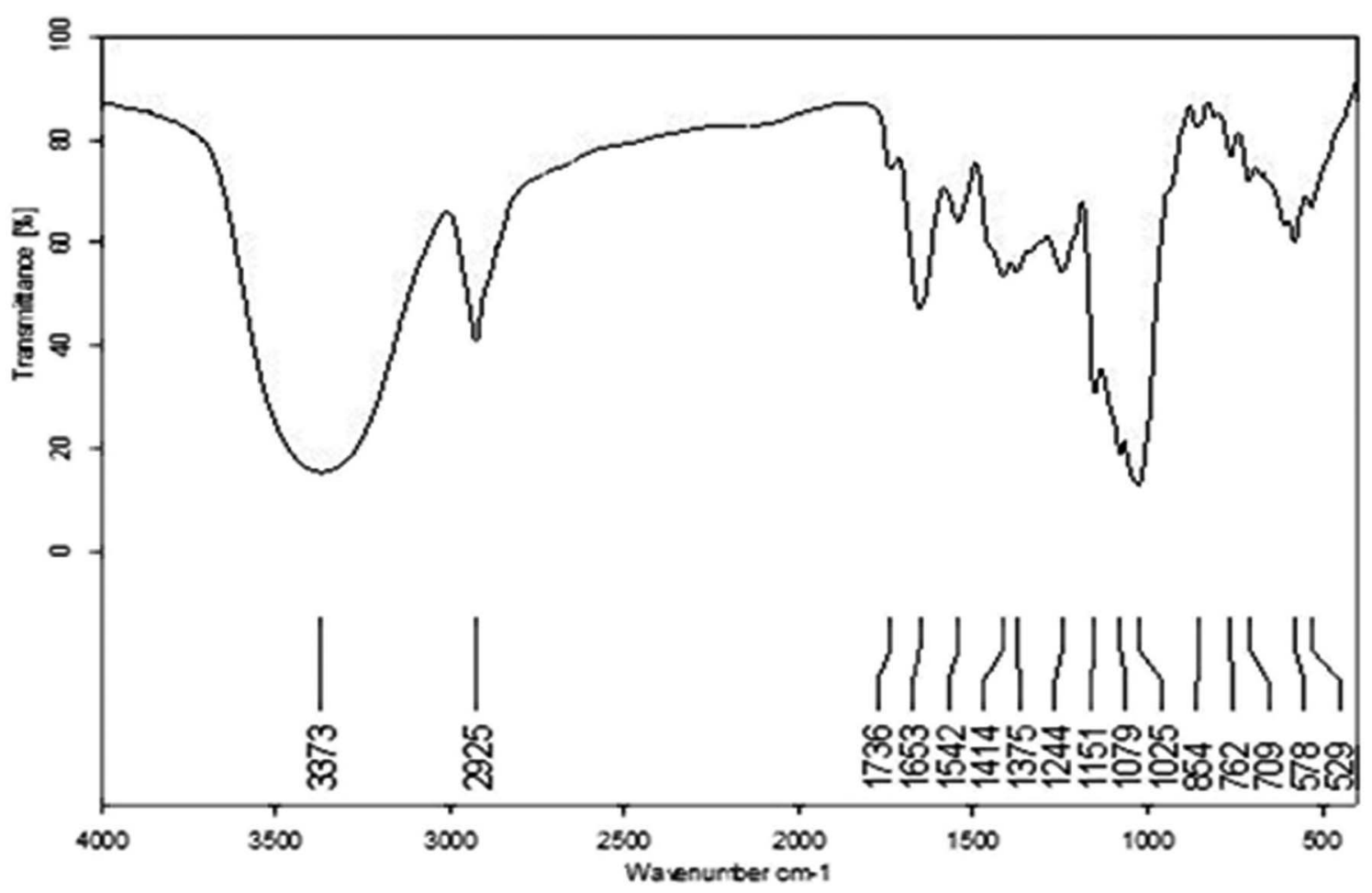

A

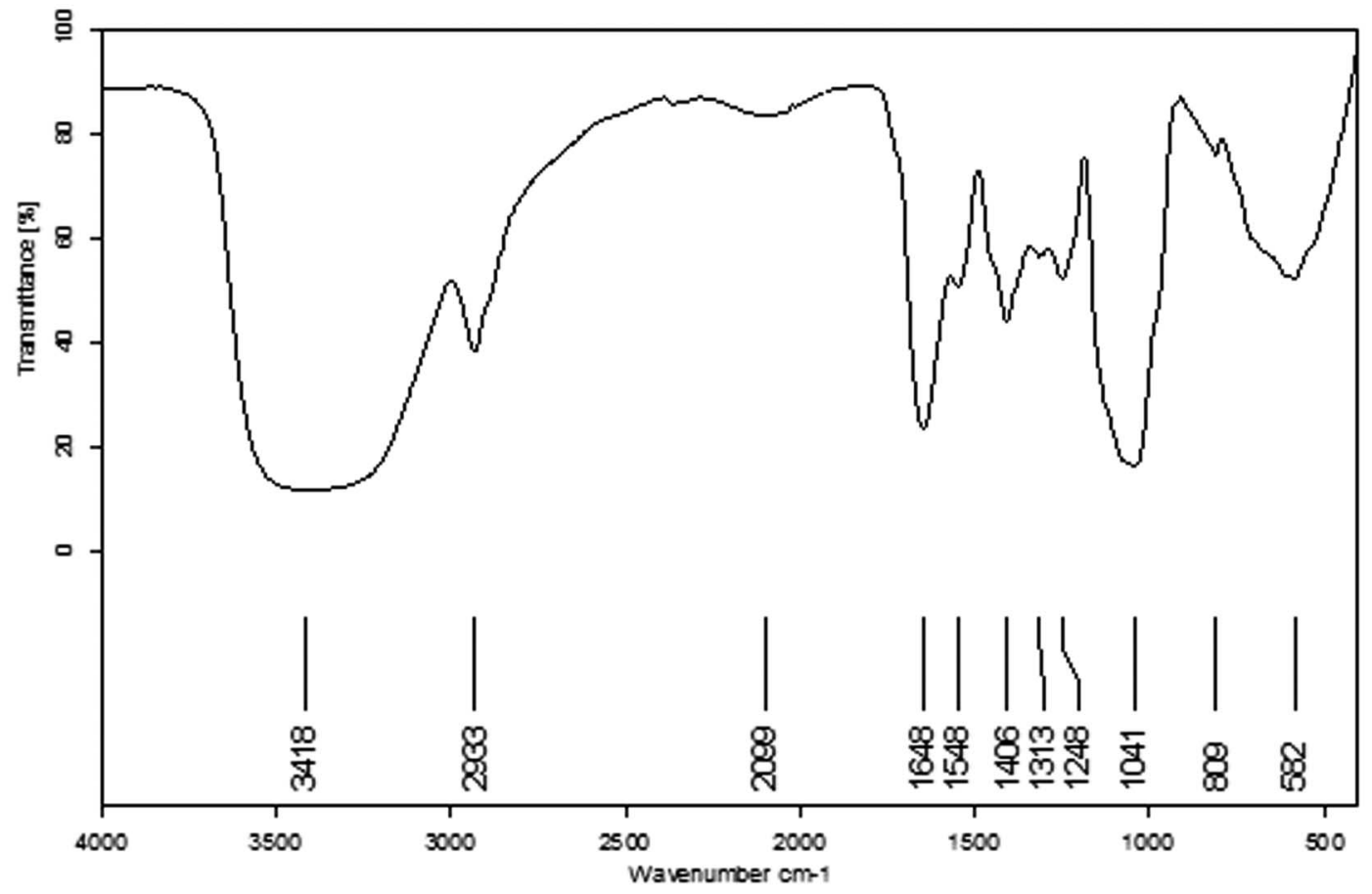

B

Fig. 4 The IR spectrum of CPS-JC (A) and CPS-YH (B).

\section{Structural analysis of CPS-JC and CPS-YH}

In TLC the hydrolysates of CPS-JC, CPS-YH and standard monosaccharides (Fig. 3A) appeared as a homogenous point, indicating that all samples displayed signs of complete acid degradation. Additionally, the location of CPS-JC and CPS-YH was similar to that of D-mannose, L-arabinose, and D-glucose, indicating the presence of these sugars. The exact monosaccharide compositions of CPS-JC and CPS-YH were further determined using GC. 
A

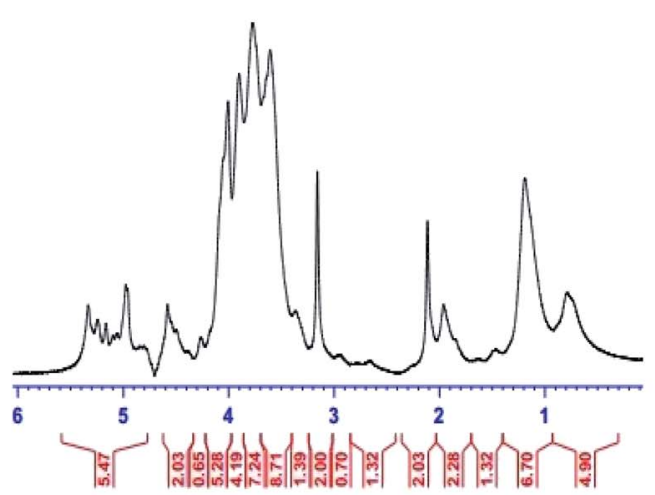

C

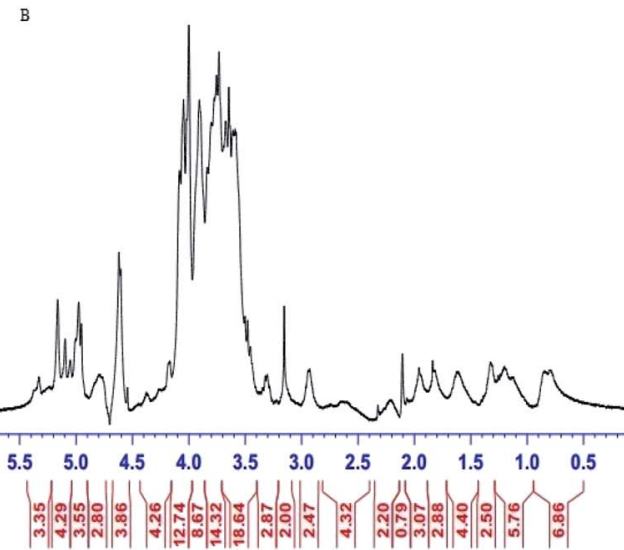

D
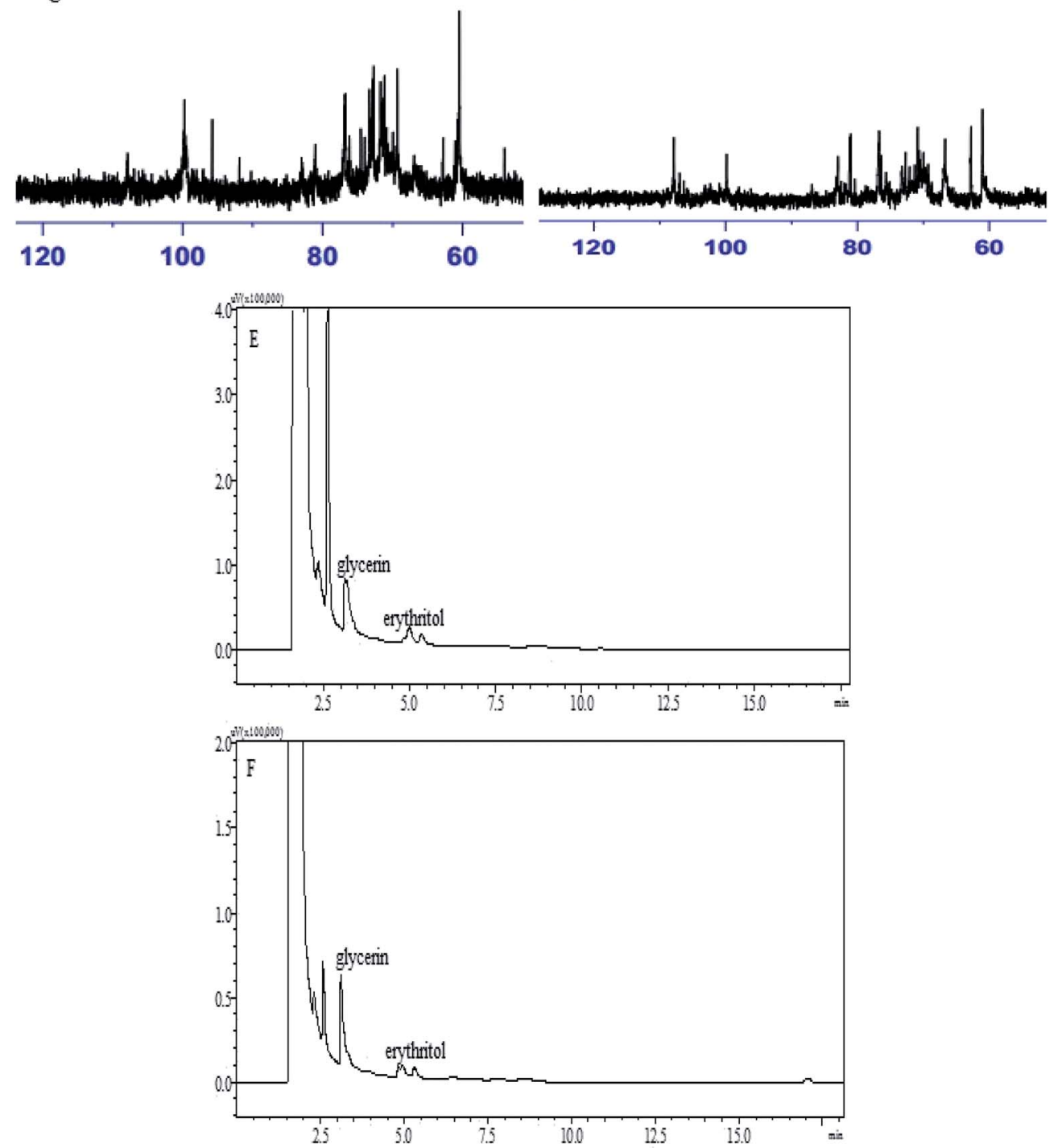

Fig. $5{ }^{1} \mathrm{H}$ NMR spectra of CPS-JC (A) and CPS-YH (B); ${ }^{13} \mathrm{C}$ NMR spectra of CPS-JC (C) and CPS-YH (D) in $\mathrm{D}_{2} \mathrm{O}$; GC chromatograms of the Smith degradation of CPS-JC (E) and CPS-YH (F).

The monosaccharide compositions of CPS-JC and CPS-YH are shown in Fig. 3B and C, respectively. The results indicated that CPS-JC and CPS-YH were both primarily composed of $\mathrm{D}^{-}$ mannose, D-glucose, and $\mathrm{D}$-galactose in the molar ratio of $1.18: 4.39: 1.30$ and $2.37: 4.56: 2.23$, respectively, with a trace amount of other sugars. 
The IR spectra for the CPS-JC and CPS-YH samples are shown in Fig. $4 \mathrm{~A}$ and B. The broad stretching peaks at $3373 \mathrm{~cm}^{-1}$ (CPSJC) and $3418 \mathrm{~cm}^{-1}$ (CPS-YH) were ascribed to hydroxyl groups with stretching vibration, while the weak absorption peaks at $2925 \mathrm{~cm}^{-1}$ (CPS-JC) and $2933 \mathrm{~cm}^{-1}$ (CPS-YH) were characteristic of $\mathrm{C}-\mathrm{H}$ stretching vibration. Additionally, the bands at $1653 \mathrm{~cm}^{-1}$ (CPS-JC) and $1648 \mathrm{~cm}^{-1}$ (CPS-YH) exhibited a strong absorption peak indicative of $\mathrm{C}=\mathrm{O}$ stretching vibration. Therefore, the three bands above were characteristic IR absorption peaks of polysaccharides. Furthermore, strong absorption in the range of $1200-1000 \mathrm{~cm}^{-1}$ was apparent, suggesting that the monosaccharides in CPS-JC and CPS-YH both displayed a pyranose ring. ${ }^{45}$ The characteristic absorption bands at $854 \mathrm{~cm}^{-1}$ indicated that CPS-JC contained $\alpha$-glycosidic linkages. However, CPS-YH exhibited no visible characteristic absorption peaks at $850 \mathrm{~cm}^{-1}$. These results suggest that CPS-JC could be a type of alpha-pyran polysaccharide. However, further study is required to establish the exact configuration of CPS-YH.

The results of NMR analysis involving ${ }^{1} \mathrm{H}-\mathrm{NMR}$ and ${ }^{13} \mathrm{C}-\mathrm{NMR}$ experiments were used to assign the chemical shifts of the sugar residues. ${ }^{46}$ The ${ }^{1} \mathrm{H}$ NMR spectrum of CPS-JC (Fig. 5A) showed that the anomeric proton resonance exceeded $4.95 \mathrm{ppm}$, and is attributed to $\alpha$-pyranose. There were three primary anomeric $\mathrm{H}$ at $\delta 5.374-\delta 4.964 \mathrm{ppm}$, indicating that CPS-JC was mainly composed of three types of sugar. Moreover, the ${ }^{13} \mathrm{C}$ NMR spectrum of CPS-JC (Fig. 5C) displayed no signal at a low field from 160-180 ppm, which indicated that no uronic acid was present. Furthermore, three anomeric C at $\delta 97-\delta 106 \mathrm{ppm}$ were evident, and the peak at $99.76 \mathrm{ppm}$ corresponded to C- 1 of $\alpha$-Dpyranose, which was in accordance with the analysis of the IR spectrum. The ${ }^{1} \mathrm{H}$ NMR spectrum (Fig. 5B) and ${ }^{13} \mathrm{C}$ NMR spectrum (Fig. 5D) of CPS-YH displayed similar signals when compared with CPS-JC. However, a weak signal at $106 \mathrm{ppm}$ indicated that $\beta$-D-glycosidic linkage might be present in CPSYH.

The periodate oxidation of CPS-JC and CPS-YH consumed $1.96 \times 10^{-4} \mathrm{~mol}$ and $2.04 \times 10^{-4} \mathrm{~mol}$ of $\mathrm{NaIO}_{4}$, respectively. No formic acid was observed, indicating the absence of $1 \rightarrow 6$ linked saccharides in the two samples. After Smith degradation analysis of the oxidation in CPS-JC (Fig. 5E) and CPS-YH (Fig. 5F), glycerol and a trace amount of erythritol were found in the degradation products using GC analysis. The molar ratio of glycerol and erythritol was $0.9164: 0.0836$ and $0.7686: 0.2314$, respectively in CPS-JC and CPS-YH. The relatively high content of glycerol was indicative of $1 \rightarrow 2$ linkage content in the main chain or branch. The substantially lower content of erythritol suggested the $1 \rightarrow 4$ linkages. The results from the periodate oxidation-Smith degradation indicated that $1 \rightarrow 4,1 \rightarrow 2$ linkages or $1 \rightarrow 3$ linkages might exist in CPS-JC and CPS-YH. These results implied that different fermentation conditions did not affect the linkage of carbohydrate chains.

Based on the TLC, GC, IR, NMR, and periodate oxidationSmith degradation results, CPS-JC and CPS-YH might contain $1 \rightarrow 4$ linked Glcp residues, as well as some $1 \rightarrow 2$ linked, $1 \rightarrow 3$ linked Glcp residues. However, CPS-JC only had $\alpha$-D-configuration, while CPS-YH had both $\alpha$-D- and $\beta$-D-configurations.

\section{Conclusion}

The results of this research indicated that the balance of environmental and nutritional conditions control the biological activities of the C. gunnii polysaccharides. In optimized conditions, the highest inhibition ratio for IPS with $\alpha$-glycosidic linkages in the S180 tumor cells reached $80 \%$. However, IPS with a trace amount of $\beta$-glycosidic linkages displayed lower activity (less than 70\%) in the same tumor cells. Additionally, the $M_{\mathrm{w}}$ of CPS-JC and CPS-YH were $8.8 \times 10^{5}$ Da and $12.68 \times$ $10^{5} \mathrm{Da}$, respectively, indicating that the IPS of $C$. gunnii with a higher $M_{\mathrm{w}}$ exhibited a higher inhibition ratio in S180 tumor cells. To conclude, the different fermentation conditions significantly affected the structure and anti-tumor activities of C. gunnii polysaccharides. The information obtained in this study will be used to examine the efficient and focused production of polysaccharides with anti-tumor properties in the submerged fermentation of Cordyceps. Further research about the inhibitory effect of polysaccharides on other tumor cells are in preparation.

\section{Conflicts of interest}

There are no conflicts to declare.

\section{Acknowledgements}

This work was financially supported by Sichuan Science and Technology Program (No. 2018ZR0283), the Open Research Subject of Key Laboratory (Research Base) of Food Biotechnology (No. szjj2017-107) and the National Natural Science Foundation of China (31871791).

\section{References}

1 Z. Y. Zhu, Y. Liu, C. L. Si, J. Yuan, Q. Lv and Y. Y. Li, Carbohydr. Polym., 2013, 92, 872-876.

2 Z. Y. Zhu, C. L. Si, Y. R. Zhong, C. M. Zhu, J. P. Zhou, A. J. Liu and Y. M. Zhang, J. Food Biochem., 2011, 35, 303-322.

3 B. Z. Zhang, P. S. Yan, H. Chen and J. He, Carbohydr. Polym., 2012, 87, 2569-2575.

4 Z. Y. Zhu, X. C. Liu, Y. L. Tang, F. Y. Dong, H. Q. Sun, L. Chen and Y. M. Zhang, J. Pharm. Biomed. Anal., 2016, 122, 494-498.

5 L. Huang, Q. Z. Li, Y. Y. Chen, X. F. Wang and X. W. Zhou, Afr. J. Microbiol. Res., 2009, 3, 957-961.

6 M. Mina, K. D. Shonkor, H. Masanori, F. Shinya and S. Akihiko, Process Biochem., 2014, 49, 181-187.

7 F. S. Reis, L. Barros, R. C. Calhelha, A. Ćirić, L. J. van Griensven, M. Soković and I. C. Ferreira, Food Chem. Toxicol., 2013, 62, 91-98.

8 L. Y. Wang, K. L. Cheong, D. T. Wu, L. Z. Meng, J. Zhao and S. P. Li, Int. J. Biol. Macromol., 2015, 79, 180-185.

9 J. Zhao, J. Xie, L. Y. Wang and S. P. Li, J. Pharm. Biomed. Anal., 2014, 87, 271-289.

10 X. L. Chen, G. H. Wu and Z. L. Huang, Int. J. Biol. Macromol., 2013, 58, 18-22. 
11 S. P. Nie, S. W. Cui, A. O. Phillips, M. Y. Xie, G. O. Phillips, S. Al-Assafd and X. L. Zhang, Carbohydr. Polym., 2011, 84, 894-899.

12 Z. Y. Zhu, N. Liu, C. L. Si, Y. Liu, L. N. Ding and J. Cheng, Carbohydr. Polym., 2012, 88, 1072-1076.

13 P. H. Leung, H. Zhao, K. P. Ho and J. Y. Wu, Food Chem., 2009, 114, 1251-1256.

14 R. M. Yu, W. Yang, L. Y. Song, C. Y. Yan, Z. Zhang and Y. Zhao, Carbohydr. Polym., 2007, 70, 430-436.

15 M. Zhang, L. Zhu, S. W. Cui, Q. Wang, T. Zhou and H. S. Shen, Int. J. Biol. Macromol., 2011, 48, 5-12.

16 L. Y. Zhao, Y. H. Dong, G. T. Chen and Q. H. Hu, Carbohydr. Polym., 2010, 80, 783-789.

17 J. S. Dixon and D. Lipkin, Anal. Chem., 1954, 26, 1092-1093. 18 L. D. Li, M. Z. Fan and Z. Z. Li, J. Microbiol., 2000, 27, 427428.

19 J. L. Casas López, J. A. Sánchez Pérez, J. M. Fernández Sevilla, F. G. Acién Fernández, E. Molina Grima and Y. Chisti, Enzyme Microb. Technol., 2003, 33, 270-277.

20 S. W. Kim, H. J. Hwang, C. P. Xu, J. M. Sung, J. W. Choi and J. W. Yun, J. Appl. Microbiol., 2003, 94, 120-126.

21 Y. W. Lin and B. H. Chiang, Process Biochem., 2008, 43, 244250.

22 A. Kai, M. Kikawa, K. Hatanaka, K. Matsuzaki, T. Mimura and Y. Kaneko, Carbohydr. Polym., 2008, 54, 381-383.

23 K. H. Shin, S. S. Lim, S. Lee, Y. S. Lee, S. H. Jung and S. Y. Cho, Phytother. Res., 2003, 17, 830-833.

24 Q. L. Huang, Y. Jin, L. N. Zhang, P. C. K. Chenung and J. F. Kennedy, Carbohydr. Polym., 2007, 70, 324-333.

25 Z. Y. Zhu, F. Y. Dong, X. C. Liu, Q. Lv, F. Liu, L. T. Chen, T. Wang, Z. Wang and Y. M. Zhang, Carbohydr. Polym., 2016, 140, 461-471.

26 Y. Wei, L. W. Wang, D. J. Wang, D. Wang, C. W. Wen, B. X. Han and Z. Ouyang, Chin. Med., 2018, 13, 1-11.

27 B. Salehi, M. Bayat, M. Dezfulian, A. Sabokbar and B. Tabaraie, Saudi J. Biol. Sci., 2018, 25, 1236-1241.
28 C. Wei, Z. Zhao, F. C. Shi and Q. L. Yong, Bioresour. Technol., 2008, 99, 3187-3194.

29 J. K. Yan, W. Q. Wang and J. Y. Wu, J. Funct. Foods, 2014, 6, 33-47.

30 X. B. Mao and J. J. Zhong, Biotechnol. Prog., 2004, 20, 14081413.

31 S. A. Barker, E. J. Bourne, M. Stacey and D. H. Whiffen, J. Chem. Soc., 1954, 1, 171-176.

32 A. Linker, L. R. Evans and G. Impallomeni, Carbohydr. Polym., 2001, 335, 45-54.

33 J. Dou, Y. H. Meng, L. Liu, J. Li, D. Y. Ren and Y. R. Guo, Int. J. Biol. Macromol., 2015, 72, 31-40.

34 S. Yang, L. Jin, X. D. Ren, J. H. Lu and Q. F. Meng, J. Food Drug Anal., 2014, 22, 468-476.

35 S. W. Zhan, J. Yang and J. Chen, Appl. Biocatal., 2004, 15, 256.

36 E. S. Lin and S. C. Sung, Int. J. Food Microbiol., 2006, 108, 182-187.

37 C. Hsieh, M. J. Tsai, T. H. Hsu, D. M. Chang and C. T. Lo, Appl. Biochem. Biotechnol., 2005, 120, 145-157.

38 M. Papagianni, Biotechnol. Adv., 2004, 22, 189-259.

39 S. W. Kim, C. P. Xu, H. J. Hwang, J. W. Choi, C. W. Kim and J. W. Yun, Biotechnol. Prog., 2003, 19, 428-435.

40 Q. F. Meng, X. Y. Lin and W. Li, Amino Acids Biotic Resour., 2005, 27, 33-35.

41 P. Xu, Z. Y. Ding, Z. Qian, C. X. Zhao and K. C. Zhang, Enzyme Microb. Technol., 2008, 42, 325-331.

42 Q. L. Huang, Y. Jin, L. N. Zhang, P. C. K. Cheung and J. F. Kennedy, Carbohydr. Polym., 2007, 70, 324-333.

43 Y. F. Peng, L. N. Zhang, F. B. Zeng and Y. X. Xu, Carbohydr. Polym., 2003, 54, 297-303.

44 J. Q. Wang, L. J. Kan, S. P. Nie, H. H. Chen, S. W. Cui, A. O. Phillips, G. O. Phillips, Y. J. Li and M. Y. Xie, LWTFood Sci. Technol., 2015, 63, 2-7.

45 Y. Zhang, M. Gu, K. Wang, Z. X. Chen, L. Q. Dai, J. Y. Liu and F. Zeng, Fitoterapia, 2010, 81, 1163-1170.

46 H. Y. Li, W. J. Mao, Y. J. Hou, Y. Gao, X. H. Qi and C. Zhao, Bioresour. Technol., 2012, 114, 414-418. 